\title{
Probing the Nanoscale with High-Speed Interferometry of an Impacting Drop
}

\author{
S. T. Thoroddsen ${ }^{1 *}$, E. Q. Li $^{1}$, I. U. Vakarelski ${ }^{1}$, K. Langley ${ }^{1}$ \\ ${ }^{1}$ King Abdullah University of Science and Technology (KAUST), Thuwal, Saudi Arabia \\ *Corresponding Author [sigurdur.thoroddsen@kaust.edu.sa]
}

\begin{abstract}
The simple phenomenon of a water drop falling onto a glass plate may seem like a trivial fluid mechanics problem. However, detailed imaging has shown that this process is highly complex and a small air-bubble is always entrapped under the drop when it makes contact with the solid. This bubble can interfere with the uniformity of spray coatings and degrade inkjet fabrication of displays etc. We will describe how we use high-speed interferometry at 5 million frames per second to understand the details of this process. As the impacting drop approaches the solid, the dynamics are characterized by a balance between the lubrication pressure in the thin air layer and the inertia of the bot-tom of the drop. This deforms the drop, forming a dimple at its bottom and making the drop touch the surface along a ring, thereby entrapping the air-layer, which is typically 1-3 $\mu \mathrm{m}$ thick. This air-layer can be highly compressed and the deceleration of the bottom of the drop can be as large as 300,000 g. We describe how the thicknessevolution of the lubricating air-layer is extracted from following the interference fringes between frames. Two-color interferometry is also used to extract absolute layer thicknesses. Finally, we identify the effects of nanometric surface roughness on the first contact of the drop with the substrate. Here we need to resolve the $100 \mathrm{~nm}$ thickness changes occurring during 200 ns intervals, requiring these state of the art high-speed cameras. Surprisingly, we see a ring of micro-bubbles marking the first contact of the drop with the glass, only for microscope slides, which have a typical roughness of $20 \mathrm{~nm}$, while such rings are absent for drop impacts onto molecularly smooth mica surfaces.
\end{abstract}

Keywords: High frame rate, interferometry, drop impact, air films

\section{INTRODUCTION}

Multiphase flows of drops are of great industrial importance, from the petrochemical industry to inkjet printing and everywhere in-between. The dynamics of these flows are often controlled by singular events, like splashing during the impact of a drop, the coalescence of two drops or the pinch-off of a drop from a nozzle ${ }^{(1)}$. These events are characterized by very rapid dynamics and tiny length scales, e.g. when the neck of the pinching drop goes to zero or the film between coalescing drops becomes exceedingly thin and ruptures, which had proven very challenging to observe experimentally until the advent of high-speed cameras. The invention of fast $\mathrm{CMOS}^{(2)}$ and in-situ image sensors ${ }^{(3-5)}$ during the last couple of decades has truly revolutionized the study of multiphase flows ${ }^{(6)}$, revealing many unexpected phenomena that were never envisioned such as the formation of a vortex street within a drop impacting on a pool surface ${ }^{(7)}$. These very fine details present a great challenge to numerical simulations, which invariably cannot resolve the finest scales. One example, important in the coarsening of emulsions, is the rupture of the thin films separating emulsion droplets. Here high-speed imaging can help formulate models to incorporate into large-scale numerical simulations of oil-water separators. Recently, high-speed imaging has been complimented by X-ray imaging ${ }^{(8)}$ and interferometry.

In what follows we review a few of our recent drop-impact experiments, where we have combined ultra-high-speed imaging with interferometry to study thin layers of air. First, we show the experimental configurations and the interferometric setup. Then we show the breakup of very thin films of air, which wrap around viscous drops impacting at low velocities on a pool surface. This is followed by the impact of a drop on a solid surface ${ }^{(9,10)}$. This canonical problem has received renewed interest since it was discovered that by reducing the air-pressure splashing can be eliminated ${ }^{(11)}$. The detailed dynamics of the air-layer under the impacting drop are therefore being subjected to renewed scrutiny.

\section{EXPERIMENTAL SETUP}

We study the air-film under a drop impacting onto a solid surface, which is, in our setup, a microscope glass slide. The thickness of the air-disc caught under the center of the drop is typically 1-3 $\mu \mathrm{m}$, making its study ideal for interferometric techniques. The imaging is done from the bottom through the glass plate, with various optical setups, depending on the particular situation. Figure 1(a,b) shows schematically these different interferometry setups used in our experiments.

Selected Papers from the 31st International Congress on High-Speed Imaging and Photonics, edited by Hiroyuki Shiraga, Takeharu Goji Etoh, Proc. of SPIE Vol. 10328, 1032818

(C) 2017 SPIE · CCC code: 0277-786X/17/\$18 - doi: 10.1117/12.2270068 

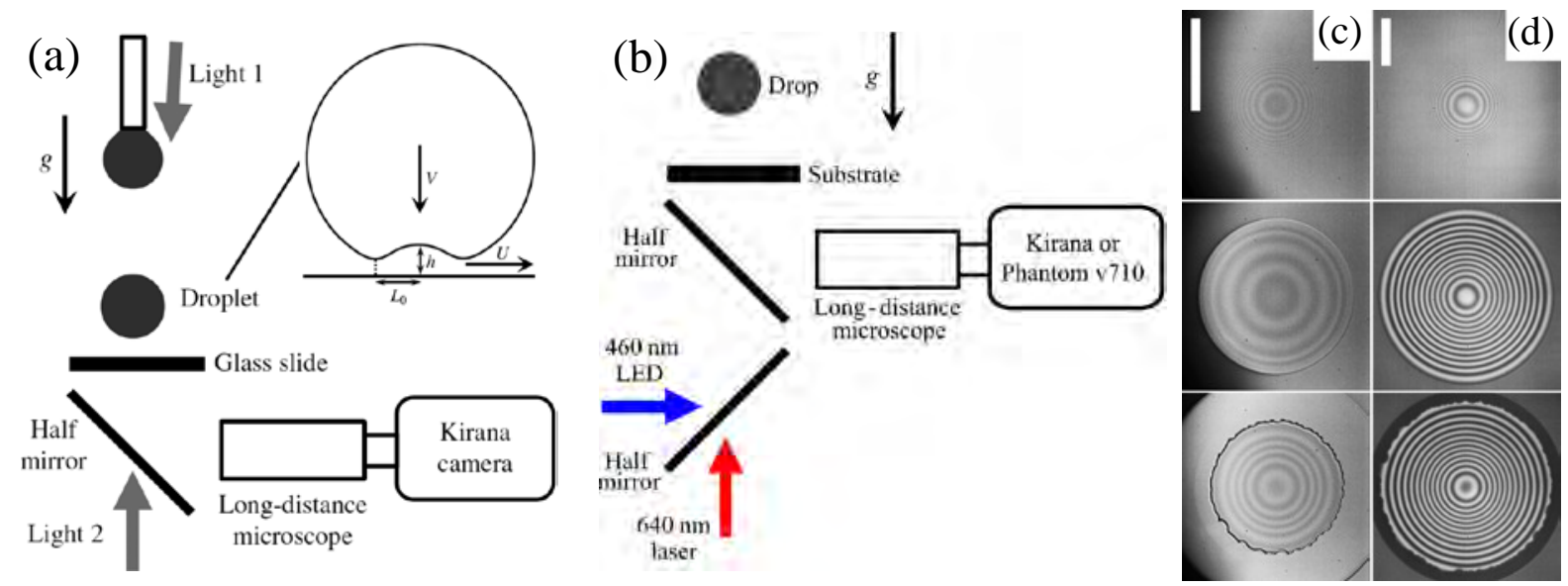

Figure 1. Optical setups used for the interferometric imaging. (a) Monochromatic light is either shone from the bottom (Light 2) through a half-silvered mirror, for reflective interferometry, or from the top (Light 1) for transmission interferometry ${ }^{(12)}$. (b) Twocolor interferometry setup, used in Langley et al. ${ }^{(15)}$. (c,d) Interference fringes under the impacting drop, from transmitted (c) and reflective (d) interferometry with monochromatic light ${ }^{(12)}$. Scale bars are both $200 \mu \mathrm{m}$ long.

We apply both reflective interferometry with the light coming from the bottom through the glass surface, as well as transmission interferometry by shining the light from the top through the drop, while the camera always images through the glass bottom plate. Transmission interferometry has the advantage of making the contact area visible, while the reflective setup produces fringes with a better signal-to-noise ratio, see Fig. 1(c,d). To further reduce noise we average the intensities around the azimuthal direction, as the fringes are axisymmetric, if the drop is axisymmetric. We have verified that the results are the same for these two setups. Interferometry with monochromatic light is most powerful in measuring changes in the thickness of a film, but it is not trivial to obtain the absolute thickness from the fringes. This can be overcome, in our context, by using two or more colors ${ }^{(13,14)}$. However, in most of our work we use a different approach. With the 5 million fps Kirana video camera ${ }^{(16)}$ we are able to follow interference fringes from one frame to the next and thereby keep track of absolute thicknesses, while using monochromatic light. This requires identifying the last fringe before the drop touches the solid surface, therefore allowing us to trace back the absolute thicknesses at earlier times, by keeping track of new fringe formations. This camera is based on in-sensor image storage ${ }^{(3,4,16)}$ and can acquire a sequence of 180 frames, which corresponds to a total clip duration of $36 \mu$ s at the highest frame-rate. This mandates the use of electronic triggering for which we use a laser/photo-diode circuit, which the drop interrupts during its free-fall. The lighting is accomplished by a separate unit of 180 laser-diodes (SI-LUX640), one for each frame, which can be independently controlled with each pulse as short as $50 \mathrm{~ns}$. The red light has wavelength of $\lambda=640 \mathrm{~nm}$ giving a thickness resolution between an adjacent dark and a bright fringe of $\lambda / 4=160 \mathrm{~nm}$. The long-distance microscope (Leica Z16 APO) has magnification up to 29.4 and can reach around $1 \mu \mathrm{m} / \mathrm{px}$ resolution even at the highest frame-rate. In limited cases we used a two colors setup (red and blue) to measure absolute thicknesses ${ }^{(15)}$, but this could only be applied up to $16 \mathrm{kfps}$ and was used to supplement the high-speed monochromatic results, as shown in sec. 3.4.

\section{RESULTS}

\subsection{Breakup of Thin Air Films Around a Drop Impacting a Deep Pool}

Besides using interferometry to measure the air-disc under an impacting drop, we have also applied this technique in order to investigate the breakup of extended air films wrapped around a drop impacting a deep pool. Such breakup of ten leaves behind a large number of small bubbles and goes by the name of Mesler entrainment ${ }^{17-19)}$. This configuration is challenging for interferometry due to the large curvature of the free surface ${ }^{(20)}$.

However, for one variant of this experiment, where one produces fine cylindrical sheets of air, which form around highly viscous drop impacting on a lower viscosity pool ${ }^{(21)}$. One such realization is shown in Figure 2, where the drop viscosity is $10,000 \mathrm{cSt}$ silicon oil and the pool is only $1 \mathrm{cSt}$. Here we study the thickness of the film along the centerline of the viscous thread. One must take account of the slope of the film with respect to the vertical, especially where the thread meets the spherical drop. Images with different magnifications are required to extract the air-layer thickness along the entire thread. Furthermore, it is intriguing that the long cylindrical sheet of air remains intact, as it should be 


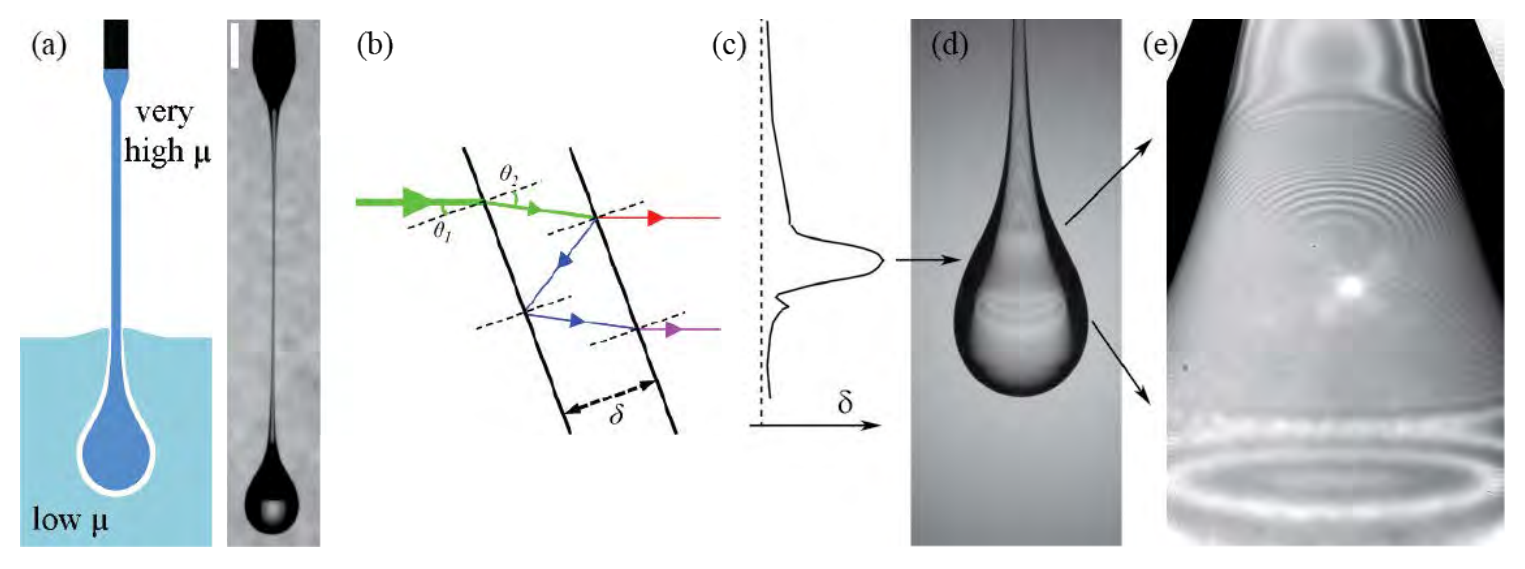

Figure 2. Interferometry of a thin film wrapped around an impacting drop, modified from Beilharz et al. ${ }^{(21)}$. (a) Schematic of experiment and entrainment of air film during impact. (b) Schematic of the interference when the free surface is not perpendicular to the lighting. Monochomatic lighting is here directed horizontally through the pool. (c) Thickness profile $\delta$ along the air-film. The maxima in the curve is about $6.5 \mu \mathrm{m}$. (d) The fringes along the drop, which is about $2.4 \mathrm{~mm}$ in diameter. In the central section the fringes change too rapidly, showing an indistinct fuzzy region. (e) Image at a larger optical magnification can separate individual fringes.

unstable to Rayleigh capillary instability of hollow cylinders. Contact across the air-layer is thereby stabilized, perhaps by static electric forces, or viscous drag within the air-layer. Our measurements indicate that these air-layers can remain intact even down to about $75 \mathrm{~nm}$ thickness. This we estimate from the size of the resulting micro-bubbles, as this thickness is below the resolution of the interferometry. The air-layer thickness near the bottom of the drop cannot be determined from this view, as there is too much diffraction. Bottom view would help in this respect ${ }^{(20)}$, but depth of focus becomes a problem when the drop moves far into the pool.

\subsection{Compressible Air-Disc under an Impacting Drop}

The early interferometric video imaging of the air-layers under impact drops ${ }^{(22-26)}$ were not time-resolved and could therefore not track the compression of the air inside the central dimple. Our time-resolved imaging has revealed new aspects of the air-layer dynamics ${ }^{(12)}$, as the bottom of the drop is deformed into a dimple, which makes first contact with the solid not at a point, but rather along a ring which entraps the air-film. Furthermore, at high impact velocities, $\sim 3$ $\mathrm{m} / \mathrm{s}$, there is significant compression of the air. Our measurements show that this compression is most likely adiabatic, not isothermal as suggested by some of the theories. With our $200 \mathrm{~ns}$ time resolution we track how the volume of the air can be compressed at the first contact by more than a factor of 10 at moderate impact velocities of $6 \mathrm{~m} / \mathrm{s}$. This is followed by a rapid vertical expansion of the air-layer, which occurs within the first $2 \mu \mathrm{s}$. This expansion phase would be entirely missed at frame-rates below $500 \mathrm{kfps}$. We point out that this large compression will change temperature of the gas and thereby the gas viscosity, which needs to be included in the lubrication theories ${ }^{(27)}$. Subsequently, the airdisc rapidly contracts into a central bubble ${ }^{(23,24)}$. This contraction into the central bubble is accurately modeled with a capillary-inertial scaling, for the low-viscosity case ${ }^{(23-25)}$. Our time-resolved measurements of the air-disc size and initial thickness have essentially verified the theoretical treatment of this problem ${ }^{(12)}$, where the basic balance is between viscous lubrication pressure within the gas in the thin air-layer and the inertia of the bottom section of the drop. This introduces a Stokes number, $S t=\mu_{g} / \rho_{l} R_{b} V$, as the controlling non-dimensional parameter. Our results also confirmed the theoretical predictions of the initial diameter of the air-disc, as well as the radial velocity of the kink in the bottom shape of the drop ${ }^{(12)}$.

\subsection{First Contact of a Drop Impacting on a Surface with Nano-metric Roughness}

Owing to the cushioning of the drop by the intervening air, the drop-bottom develops a dimple and it contacts the solid surface, not at a point, but along a ring, thus entrapping the thin air-disc as was shown in Fig. 1(c,d) and in Fig. 3(a). Often this first contact leaves a distinct dark region, as shown in Fig. 3(b). This was observed in early imaging ${ }^{(24)}$ and speculated to be due to micro-bubbles. With the high-speed interferometry we have indeed verified this hypothesis and shown that the roughness of the solid plays a key role in this entrapment of the ring of micro-bubbles ${ }^{(26)}$. Figure 3(c) and (d) compare the first contact between a regular microscope slide with $20 \mathrm{~nm}$ roughness elements and a molecularly 

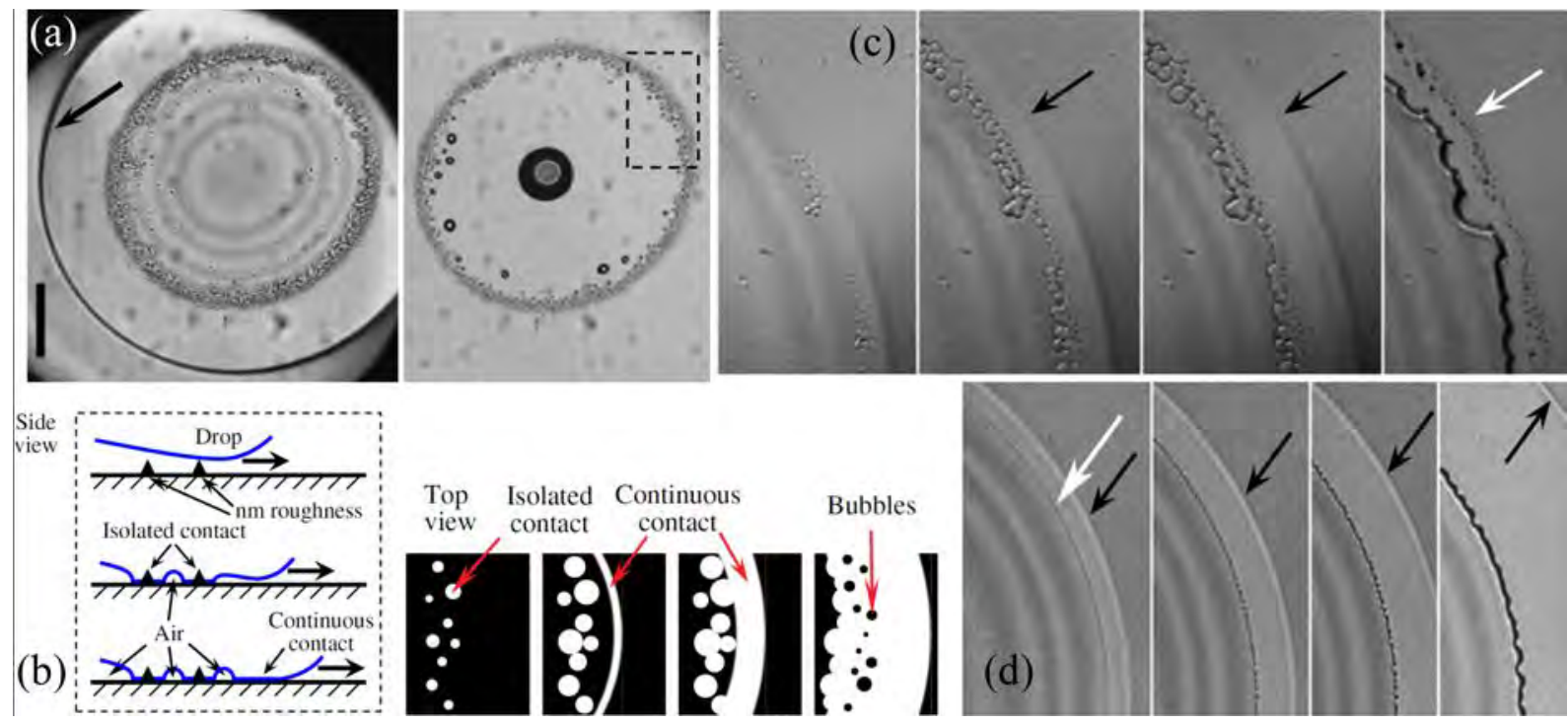

Figure 3. The first contact of an impacting drop on a smooth solid surface. (a) Interferometry showing fringes within the entrapped disc at $2 \mu$ s after first contact with a glass microscope slide. Second panel shown at $400 \mu$ s later after the air-layer has contracted into a central bubble, with a ring of micro-bubbles left at the location of the original contact. Scale bar is $200 \mu$ m. Rectangular dotted outline shows the approximate region in panels (c,d). (b) Schematic of the first contact of the drop and the micro-bubble entrapment mechanism. (c) Comparison of the first contact of a water drop with a regular microscope glass slide and (d) impact on a molecularly smooth freshly cleaved mica surface. Black arrows point at the continuous outer contact line with the solid. White arrow points at the line of micro-bubbles (top) and inner contact line (bottom). Frames are spaced by $200 \mathrm{~ns}$, except the last frame in (c), which is $1.8 \mu$ s later. Modified from Li et al. ${ }^{(26)}$.

smooth freshly cleaved mica surface. These surface roughnesses are determined with an Atomic Force Microscope (AFM) and are quite small for the microscope glass, which is thoroughly cleaned before each impact. The impact in Fig. 3(a) is on glass that has not been cleaned, resulting in a very pronounced micro-bubble ring. For the "rougher" glass surface, in Fig. 3(c), the formation of the micro-bubble ring is as follows: When the air-layer thickness is less than $100 \mathrm{~nm}$ we see localized contacts appear just before the continuous contact line is established. This mechanism is shown schematically in Fig. 3(b). When these localized contacts meet the continuous wetted section, we get the entrapment of the micro-bubbles. Similar entrapment mechanism has been observed for slightly higher viscosity cases during the lamellar spreading ${ }^{(28-30)}$, but never before during the first contact of a drop. On the other hand when using the mica surface (Fig. 3d), we do not see any ring of micro-bubbles, supporting the role of surface roughness in the micro-bubble entrapment during the very first contact.

In separate experiments we replaced the solid surface with an extremely viscous liquid, i.e. silicon oil with dynamic viscosity of 20 million cSt. Here we see no initial bubble ring, as the liquid surface should in principle not have any roughness. However, in these water/silicon oil impacts, the contracting triple-line develops azimuthal instabilities and bubbles are entrapped by an entirely different mechanism. Finally, in this experiment we showed that for very low impact velocity the water drop can glide on the air-layer without initial contact between the two liquids. The breakup of this thin air-layer was observed with a spatial resolution of $0.6 \mu \mathrm{m} / \mathrm{px}$ and a time resolution of $200 \mathrm{~ns}$, showing the details of this breakup process in unprecedented detail ${ }^{(26)}$. This breakup leaves behind a wide axisymmetric band of micro-bubbles (see their figure 5).

\subsection{Gliding on a Thin Film of Air}

For the impact of very viscous drops, the surface deformations are quite different from that of water drops. Here the kink in the free surface, which makes the first contact with the solid ${ }^{(12,31)}$, is greatly damped and the drop does not make contact along a ring, but rather it glides on a thin air-layer ${ }^{(15)}$. This extended air-layer thins until it ruptures at numerous random locations, as is shown in Fig. 4(a). Skating on a layer of air has been suggested for low-viscosity drops ${ }^{(31-33)}$, but only realized for very low impact velocities ${ }^{(15)}$. In those studies a critical impact Weber numbers has been identified as 4 , below which the drop can bounce without contacting the solid ${ }^{(33)}$. This can even occur for hydrophilic solid 

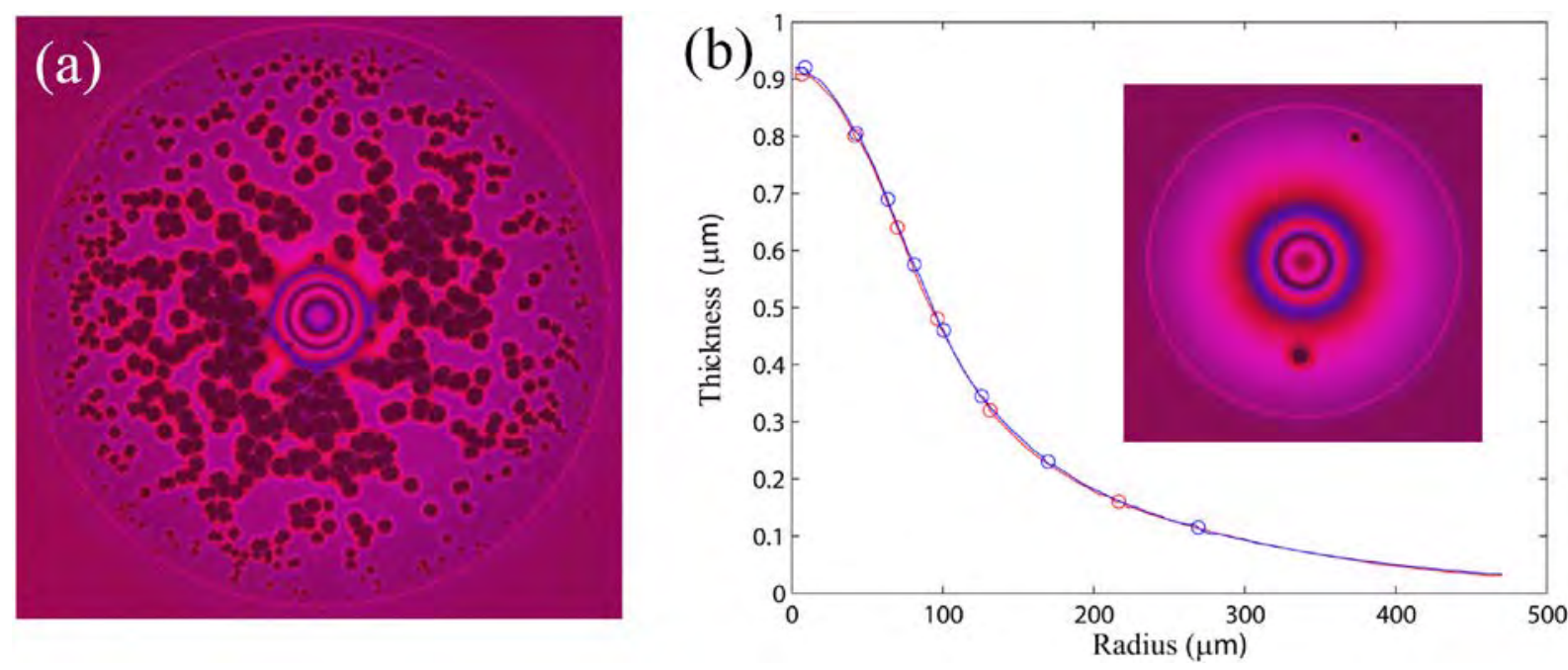

Figure 4. Two-color interferometric imaging of the thin air-layer under an impacting very viscous drop, with kinematic viscosity of $10,000 \mathrm{cSt}$. (a) The drop glides on a thin air-layer which ruptures at numerous random locations, shown by the dark spots. The drop impacts at $2.8 \mathrm{~m} / \mathrm{s}$ and the diameter of the outer edge of the thin retion is $1180 \mu \mathrm{m}$. (b) The air-layer thickness in the dimple under the center of the drop at earlier times, now impacting at $1.2 \mathrm{~m} / \mathrm{s}$. The thickness profiles are determined independently from the red and blue colors. Two local ruptures have formed. Modified from Langley et al. ${ }^{(15)}$.

surfaces $^{(33,34)}$. In contrast, for our highly viscous drops, gliding is observed for much higher impact velocities ${ }^{(15)}$. It is not obvious that these localized ruptures occur for a layer thickness less than $140 \mathrm{~nm}$ and to verify this we needed to per-form dual-color reflective interferometry, using the setup shown in Fig. 1(b). Figure 4(b) confirms this fact, i.e. that the last bright fringe before contact with the solid, in the inset, is $160 \mathrm{~nm}$ for the red light. Using this result we can compare the thickness derived from the two independent measurements (red and blue) and we see very good agreement. Here the circular symbols indicate the location of maxima/minima in the intensities, whereas the continuous curves are based on the $\sin ^{2} \delta$ intensity interpolations between the adjacent extrema in the fringes. Here the two curves deviate by less than $20 \mathrm{~nm}$ from each other. Knowing the minimum thickness fringe, we can then revert back to the monochromatic 200 ns interferometry to track the temporal evolution of the thickness long before contact. It is worth noting that the dimple for this very viscous drop in Fig. 4(b) is less than one micron thick, but its radial extent is 500 times larger. This highlights that drop deformation is strongly reduced by the viscous forces within the liquid.

\section{SUMMARY}

Herein we have highlighted our work with high-speed interferometry aimed at understanding the first contact of a drop impacting a solid surface. This technique has successfully revealed new dynamics of the entrapped air-layer and the next target is the origin of splashing, occurring at the outer edge of the liquid contact ${ }^{(35)}$. This will require absolute layer thickness measurements as the fringes are too closely spaced at the contact line. In principle there is nothing fundamental preventing two-color measurements at the highest frame-rates, except the intensity of the illumination at two different colors, which can be overcome with specialized pulsed illuminations and a color camera, or two monochrome cameras with the appropriate filters. This appears to be only a matter of time.

\section{REFERENCES}

[1] Eggers, J. "Nonlinear dynamics and breakup of free-surface flows.” Rev. Mod. Phys., 69, 865 (1997).

[2] Etoh T. G. "High speed camera of 4500 pps.” J. Inst. Telev. Eng. Jpn. 45, 543-545 (1992). (In Japanese).

[3] Kosonocky, W. F., Yang, G., Ye, C., Kabra, R.K., Xie, L. et al. “360 × 360-element very-high frame-rate burst-image sensor.” Dig. Tech. Pap. IEEE Int. Solid State Circuits Conf., 39, 182-183 (1996).

[4] Etoh T. G., Poggemann, D., Kreider, G., Mutoh, H., Theuwissen, A. J. P. , et al. "An image sensor which captures 100 consecutive frames at 1000000 frames/s." IEEE Trans. Electron. Devices, 50, 144-151 (2003).

[5] Etoh, T. G., Mutoh, H. "An image sensor of 1 Mfps with photon counting sensitivity.” Proc. 26th Int. Conf. High Speed Photogr. Photonics, 5580, 301-307. Bellingham, WA: SPIE (2005). 
[6] Thoroddsen, S. T., Etoh, T. G., Takehara, K., “High-speed imaging of drops and bubbles,” Ann. Rev. Fluid Mech., 40, 257-285 (2008).

[7] Thoraval. M.-J., Takehara, K., Etoh, T. G., Popinet, S., Ray, P. et al. "Von Karman vortex street within an impacting drop.”

Phys. Rev. Lett., 108, 264506 (2012).

[8] Zhang, L. V., Toole, J., Fezzaa, K., Deegan, R. D. “Evolution of the ejecta sheet from the impact of a drop with a deep pool.” J. Fluid Mech., 690, 5-15 (2012).

[9] Yarin, A. L. "Drop impact dynamics: splashing, spreading, receding, bouncing ...,” Annu. Rev. Fluid Mech., 38, 159-192 (2006).

[10] Josserand, C., Thoroddsen, S. T., "Drop impact on a solid surface.” Ann. Rev. Fluid Mech., 48, 365-391 (2016).

[11] Xu, L., Zhang, W. W., Nagel, S. R. "Drop splashing on a dry smooth surface.” Phys. Rev. Lett., 94, 184505 (2005).

[12] Li, E. Q., Thoroddsen, S. T., "Time-resolved imaging of a compressible air disc under a drop impacting on a solid surface.” $J$. Fluid Mech., 780, 636-648 (2015).

[13] de Ruiter, J., Mugele, F., van den Ende, D. “Air cushioning in droplet impact. II: Experimental characterization of the air film evolution,” Phys. Fluids, 27, 012104 (2015).

[14] van der Veen, R. C. A., Tran, T., Lohse, D., Sun, C., "Direct measurements of air layer profiles under impacting droplets using high-speed color interferometry.” Phys. Rev. E, 85, 026315 (2012).

[15] Langley, K., Li, E. Q., Thoroddsen, S. T., “Impact of ultra-viscous drops: air-film gliding and extreme wetting.” Accepted for publication in J. Fluid Mech.

[16] Crooks, J., Marsh, B., Turchetta, R., Taylor, K., Chan, W., Lahav, A., Fenigstein, A. "Kirana: a solid-state megapixel uCMOS image sensor for ultrahigh speed imaging.” Proc. SPIE, 8659, 865903 (2013).

[17] Sigler, J., Mesler, R. "The behaviour of the gas film formed upon drop impact with a liquid surface.” J. Colloid Interface Sci., 134 (2), 459-474 (1990).

[18] Saylor, J. R., Bounds, G. D., "Experimental study of the role of the Weber and capillary numbers on Mesler entrainment." AIChE J. doi:10.1002/aic.13764 (2012).

[19] Thoroddsen, S. T., Thoraval, M.-J., Takehara, K., Etoh, T. G., "Micro-bubble morphologies following drop impacts onto a pool surface.” J. Fluid Mech., 708, 469-479 (2012).

[20] Tran, T., De Maleprade, H., Sun, C., Lphse, D. “Air entrainment during impact of droplets on liquid surfaces.” J. Fluid Mech., 726, R3 (2013).

[21] Beilharz, D., Guyon, A., Li, E. Q., Thoraval, M.-J., Thoroddsen, S. T., “Antibubbles and fine cylindrical sheets of air”. J. Fluid Mech., 779, 87-115 (2015).

[22] Driscoll, M. M., Nagel, S. R., "Ultrafast interference imaging of air in splashing dynamics.” Phys. Rev. Lett., 107, 154502 (2011).

[23] Liu, Y., Tan, P., Xu, L., “Compressible air entrapment in high-speed drop impacts on solid surfaces.” J. Fluid Mech., 716, R9 (2013).

[24] Thoroddsen, S. T., Etoh, T. G., Takehara, K., Ootsuka, N., Hatsuki, Y., “The air-bubble entrapped under a drop impacting on a solid surface.” J. Fluid Mech., 545, 203-212 (2005).

[25] Lee, J. S., Weon, B. M., Je, J. H., Fezzaa, K. “How does an air film evolve into a bubble during drop impact?” Phys. Rev. Lett., 109, 204501 (2012).

[26] Li, E. Q., Vakarelski, I. U., Thoroddsen, S. T., “Probing the nanoscale: the first contact of an impacting drop.” J. Fluid Mech., 785, R2 (2015).

[27] Hicks, P. D., Purvis, R., “Liquid-solid impacts with compressible gas cushioning.” J. Fluid Mech., 735, 120-149 (2013).

[28] Thoroddsen, S. T., Takehara, K., Etoh, T. G., "Bubble entrapment through topological change.” Phys. Fluids, 22, 051701 (2010).

[29] Driscoll, M. M., Stevens, C. S., Nagel, S. R. Thin film formation during splashing of viscous liquids. Phys. Rev. E, 83, 036302 (2010).

[30] Palacios, J., Hernandez, J., Gomez, P., Zanzi, C., Lopez, J., “On the impact of viscous drops onto dry smooth surfaces,” Exp. Fluids, 52, 1449-1463 (2012).

[31] Mandre, S., Mani, M., Brenner, M. P., "Precursors to splashing of liquid droplets on a solid surface”. Phys. Rev. Lett., 102, 134502 (2009).

[32] Kolinski, J. M., Rubinstein, S. M., Mandre, S., Brenner, M. P., Weitz, D. A., Mahadevan, L., "Skating on a film of air: drops impacting on a surface.” Phys. Rev. Lett., 108, 074503 (2012).

[33] de Ruiter, J., Lagraauw, R., van den Ende, D, Mugele, F., "Wettability-independent bouncing on flat surfaces mediated by thin air films,” Nat. Phys., 11, 48-53 (2015).

[34] Kolinski, J. M., Mahadevan, L., Rubinstein, S. M., “Drops can bounce from perfectly hydrophilic surfaces.” Euro. Phys. Lett., 108, 24001 (2014).

[35] Thoroddsen, S. T., Takehara, K., Etoh, T. G., “Micro-splashing by drop impacts.” J. Fluid Mech., 706, 560-570 (2012). 